\title{
Cancer-associated fibroblast-secreted exosomal miR-423-5p promotes chemotherapy resistance in prostate cancer by targeting GREM2 through the TGF- $\beta$ signaling pathway
}

\author{
Guang Shan ${ }^{1,2}$, Juan $\mathrm{Gu}^{2,3}$, Daoping Zhou ${ }^{4}$, Lingxun Li', Wei Cheng ${ }^{4}$, Yueping Wang ${ }^{2,4,5}$, Tian Tang ${ }^{2,6}$ and \\ Xuedong Wang ${ }^{2,4}$
}

\begin{abstract}
Therapeutic failure in prostate cancer $(P C)$ is believed to result from its unusually invasive and metastatic nature. Cancer-associated fibroblasts (CAFs) are essential in the tumor microenvironment. We intended to study the role of CAF-derived exosomes in the context of PC and the potential regulatory mechanism associated with miR-423-5p and GREM2. CAF-derived exosomes decreased the chemosensitivity of parental PC cells and enhanced the drug resistance of drug-resistant cells. PC-associated fibroblast-derived exosomes carrying miR-423-5p increased the resistance of PC to taxane by inhibiting GREM2 through the TGF- $\beta$ pathway. Inhibition of the TGF- $\beta$ pathway partially reversed the increased drug resistance in PC cells induced by CAF-derived exosomes. Inhibition of miR-423-5p enhanced the drug sensitivity of PC cells in vivo. We showed that CAF-secreted exosomal miR-423-5p promoted chemotherapy resistance in PC by targeting GREM2 through the TGF- $\beta$ pathway. This study may allow the development of novel approaches for $P C$.
\end{abstract}

\section{Introduction}

Prostate cancer $(\mathrm{PC})$ is the $2^{\text {nd }}$ most common cancer and the $5^{\text {th }}$ most common cause of death ${ }^{1}$. Statistics show that there are 1.6 million males with $\mathrm{PC}$, and 366,000 males die from PC each year ${ }^{2}$. Many factors, including age, race, and family heritability, have been associated with PC development ${ }^{3}$. Based on the severity of this disease, current treatment approaches for PC include hormones, bone-directed treatment, therapeutic vaccines, radiation, and surgery ${ }^{4}$. Although these treatments can significantly delay or inhibit PC progression, chemotherapy resistance usually leads to death ${ }^{5}$. Cancer-associated

\footnotetext{
Correspondence: Xuedong Wang (wxd729@126.com)

${ }^{1}$ Department of Urology, RenMin Hospital of Wuhan University, Wuhan 430060 Hubei, China

2Department of Medical Laboratory Science, The Fifth People's Hospital of

Wuxi, Nanjing Medical University, Wuxi 214000 Jiangsu, China

Full list of author information is available at the end of the article

These authors contributed equally: Guang Shan, Juan Gu
}

fibroblasts (CAFs) are dominant in the tumor microenvironment because of their function in promoting tumor progression ${ }^{6}$. Additionally, CAFs act as regulators of the immune system by secreting transforming growth factor- $\beta$ (TGF- $\beta$ ) and generating conditions under which antitumor immune responses are weakened ${ }^{7}$. Furthermore, CAF-derived exosomes seemed to be an important trigger of tumor progression ${ }^{8}$.

Exosomes are ideal targets for the treatment of cancer because of their small sizes and multifunctional effects on cells ${ }^{9}$. The exact effects of exosomes on the promotion or inhibition of PC have been discussed ${ }^{10}$. Exosomes carrying proteins, messenger RNAs (mRNAs), and microRNAs (miRs) can be transferred between cells ${ }^{11}$, and miRs can regulate gene expression through degradation or suppression of target miRNAs ${ }^{12}$. In addition, the effects of miRs on PC have been discussed, and exosomal miRs are regarded as markers for $\mathrm{PC}^{13,14}$. miR-423-5p could result 
in the occurrence of malignant types and temozolomide resistance in glioblastoma ${ }^{15}$ and promote autophagy of hepatocellular carcinoma cells ${ }^{16}$. One recent report showed that exosomal miR-423-5p could promote tumor growth and metastasis by targeting Sufu ${ }^{17}$.

A microarray-based analysis in the present study showed that GREM2 was implicated in PC. GREM2, a DAN family member, is directly associated with cancers via its interactions with bone morphogenetic proteins ${ }^{18}$. However, the role of GREM2 in PC and the effect of CAF-derived exosomes on chemotherapy resistance in $\mathrm{PC}$ remain unclear. Therefore, we intended to investigate exosomal delivery within the PC microenvironment with CAFs. The abovementioned literature prompted us to surmise that CAF exosomes combined with miR-423-5p may exert effects on PC via the GREM2 and TGF- $\beta$ pathways.

\section{Materials and methods}

\section{Bioinformatics analysis}

The PC drug resistance chip GSE64012 was obtained through the GEO database (https://www.ncbi.nlm.nih. gov/) and contained 3 taxane-sensitive samples and 9 taxane-resistant samples. Using sensitive samples as a control, we used the $\mathrm{R}$ language "limma" package to analyze the differences to obtain significantly differentially expressed genes with $|\operatorname{logFC}|>2$ and $P<0.05$ as the screening criteria. The differentially expressed genes were analyzed by KEGG pathway enrichment analysis (https:// www.kegg.jp/kegg/) to obtain biological process entries for gene enrichment. The TargetScan database (http:// www.targetscan.org/vert_72/) and the miRSearch database (https://www.exiqon.com/miRSearch) were used to predict the upstream miRNAs of the target genes, the EVmiRNA database (http://bioinfolife.hust.edu.cn/ EVmiRNA\#!/browse) was used to obtain miRNAs that were highly expressed in extracellular vesicles (EVs), and then, the intersecting miRNAs were obtained. Finally, the StarBase database (http://starbase.sysu.edu.cn/index.php) was used to analyze the expression of target miRNAs in PC samples originating from TCGA and the correlation between miRNAs and target genes.

\section{Isolation of CAF-derived exosomes}

Exosomes extracted from CAF culture medium were cultured in DMEM-F12 (Sigma-Aldrich, Merck KGaA, Darmstadt, Germany) with 10\% Exo-fetal bovine serum (FBS) (FBS depleted of exosomes, SBI, Mountain View, California, USA) and $1 \times$ antibiotic-antimycotics (Life Technologies, Gaithersburg, MD, USA). After centrifugation, the supernatants were collected and added to sterile tubes containing ExoQuick-TC ${ }^{\mathrm{TM}}$ Exosome Isolation Reagent (SBI) with $2 \mathrm{~mL}$ of ExoQuick-TC ${ }^{\mathrm{TM}}$ solution for every $10 \mathrm{~mL}$ of medium, followed by agitation. Then, the tubes were stored at $4{ }^{\circ} \mathrm{C} \mathrm{O} / \mathrm{N}$ for at least $12 \mathrm{~h}$. The next day, the exosomes were centrifuged at $1500 \mathrm{~g}$ for $30 \mathrm{~min}$ and at $1500 \mathrm{~g}$ for $5 \mathrm{~min}$ at $4{ }^{\circ} \mathrm{C}$ to obtain white/ beige exosomal pellets.

CAFs were treated with miR-423-5p inhibitor or scramble inhibitor (10 nM, Tebu-Bio, San Diego, CA, USA) with Oligofectamine (Invitrogen, Carlsbad, CA, USA). Six hours later, the cells were washed, and the culture media were renewed with fresh DMEM-F12 1\% A/A supplemented with 10\% Exo-FBS. Following a 1-day incubation, culture media were harvested for exosome preparation. When incubating recipient cells (LN-CaP, $22 \mathrm{Rv}-1$ or C4-2) with exosomes, we routinely included these cells in the medium at a concentration of approximately $1 \times 10^{9}$ particles $/ \mathrm{mL}$.

\section{Immunofluorescence}

CAFs were fixed, permeabilized, and labeled with rabbit anti-vimentin, $\alpha$-SMA, and FAP mAb (Abcam), followed by amplification with FITC goat anti-rabbit antibodies and Alexa 488 goat anti-FITC antibodies (Abcam). Next, nuclei were stained with DAPI, and a laser scanning confocal microscope (FV1000, Leica) was used for observation.

\section{Characterization of exosomes}

The morphology of the exosomes was observed using a transmission electron microscope (Japan, Hitachi 7650). In addition, particle size was determined using dynamic light scattering (Malvern Instruments, Malvern, UK).

\section{Cell treatment}

The human PC cell lines LN-CaP, 22Rv-1 and C4-2 were provided by ATCC (Manassas, VA, USA). Docetaxelresistant $\mathrm{LN}-\mathrm{CaP}$ (LN-CaP/DTX) cells, taxane-resistant $22 \mathrm{Rv}-1$ (22Rv-1/TAX) cells and bicalutamide-resistant C42 (C4-2/BCT) cells were prepared by continuous exposure of the parental drug-sensitive $\mathrm{LN}-\mathrm{CaP}, 22 \mathrm{Rv}-1$ and $\mathrm{C} 4-2$ cells to gradient concentrations of drugs (Sigma-Aldrich). All cells were cultured in RPMI 1640 medium with $10 \%$ FBS, $100 \mathrm{U} / \mathrm{mL}$ penicillin and $100 \mathrm{mg} / \mathrm{mL}$ streptomycin (Invitrogen) with $5 \% \mathrm{CO}_{2}$ at $37^{\circ} \mathrm{C}$.

According to the instructions of the lentiviral packaging kit (Open Biosystems), lentivirus-carrying hsa-miR-423-5p or hsa-miR-negative control and hsa-GREM2 or empty vector was packaged in human embryonic kidney $293 \mathrm{~T}$ cells and harvested from the supernatant. Stable cell lines were established by infecting $22 \mathrm{Rv}-1 / \mathrm{TAX}$ cells with lentivirus.

\section{Reveres transcription quantitative polymerase chain reaction (RT-qPCR)}

Total RNA was isolated with TRIzol (Invitrogen) and reverse transcribed into cDNA using a Transcriptor First Strand cDNA Synthesis Kit (Toyobo, Osaka, Japan). Next, quantitative real-time PCR was performed on an 
Table 1 Primer sequences.

\begin{tabular}{lll}
\hline Sequences & Forward & Reverse \\
\hline miR-423-5p & GCCTGAGGGGCAGAGAGC & CCACGTGTCGTGGAGTC \\
GREM2 & TTCCCTGTCCTTGTTCCTG & TGCACCAGTCACTCTTGAGG \\
TGF- $\beta$ & CTCGCCGCGCTCTACCTACCTA & ATGAGCCATTCGCAGTTTCACTGTA \\
U6 & GCGCGTCGTGAAGCGTTC & GTGCAGGGTCCGAGGT \\
GAPDH & AACGACCCCTTCATTGAC & TCCACGACATACTCAGCAC \\
\hline
\end{tabular}

ABI7500 system with a SYBR Green (Thermo Fisher Scientific, USA). The PCR system contained $10 \mu \mathrm{L}$ of $2 \times$ real-time PCR buffer (including $3 \mathrm{mmol} / \mathrm{L} \mathrm{Mg}^{2+}$, $0.2 \mathrm{mmol} / \mathrm{L} \mathrm{dNTP}$ and SYBR Green I fluorescent dyes), forward and reverse specific primer sets $(5 \mathrm{mmol} / \mu \mathrm{L}$, $0.32 \mu \mathrm{L})$, Taq DNA polymerase $\left(5 \times 10^{6} \mathrm{U} / \mathrm{L}, 0.2 \mu \mathrm{L}\right)$, cDNA template $(2 \mu \mathrm{L})$, and RNase-free ultrapure water to reach a volume of $20 \mu \mathrm{L}$. GAPDH was used as the internal control of GREM2 and TGF- $\beta$. For quantitation of miR155, U6 was used as the internal control. The relative expression was quantified by normalization using the $2^{-\Delta \Delta C t}$ method. The primers are exhibited in Table 1 .

\section{Western blot analysis}

Western blot analysis was conducted as previously reported $^{19}$. The following primary antibodies were used: anti-GREM2 (1:1000, ab228736, Abcam, NY, USA) and anti-TGF- $\beta$ (1:1000, ab92486, Abcam), as well as horseradish peroxidase-conjugated secondary antibodies (1:1000, ab150117, Abcam), with anti- $\beta$-actin antibodies (ab179467, Abcam) as the control. The membrane was developed via the Super Signal West Pico Kit.

\section{In vitro cytotoxicity tests}

LN-CaP, 22Rv-1 and C4- 2 cells were cultivated at $8 \times$ $10^{3}$ cells/well in 96-well plates for $4 \mathrm{~h}$. Then, docetaxel (serial dilution, from 0 to $5 \mu \mathrm{M}$ )/taxane (serial dilution, from 0 to $10 \mu \mathrm{M}$ )/bicalutamide (serial dilution, from 0 to $10 \mu \mathrm{M}$ ) was added to the cells for incubation for $72 \mathrm{~h}$. Docetaxel, taxane and bicalutamide were purchased from Sigma-Aldrich Company (USA).

\section{Cell Counting Kit-8 (CCK-8) assay}

LN-CaP, 22Rv-1 and C4- 2 cells $\left(2.5 \times 10^{3}\right.$ cells $)$ were added to 96-well plates and treated with $10 \mu \mathrm{L}$ CCK-8 (Dojindo, Japan) for $2 \mathrm{~h}$. The proliferation rates were evaluated at $0,24,48$, and $72 \mathrm{~h}$.

\section{Colony formation assay}

A colony formation assay was utilized to measure the colony formation ability and the chemosensitivity of the PC cells to docetaxel, taxane and bicalutamide. Cells $(1 \times$
$10^{3}$ cells/well) were seeded in 6-well plates and then exposed to docetaxel, taxane and bicalutamide for $24 \mathrm{~h}$. Then, the cells were cultured for $8 \mathrm{~d}$ at $37^{\circ} \mathrm{C}$. Next, the cells were fixed in 10\% formaldehyde for $40 \mathrm{~min}$ and stained in $0.1 \%$ crystal viola for $20 \mathrm{~min}$. Finally, the colonies were counted.

\section{Flow cytometry}

Cell apoptosis was detected using a FITC Annexin V Apoptosis Detection Kit (BD Biosciences, San Jose, CA, USA $)^{20}$. Apoptotic cells were detected with a fluorescence-activated cell-sorting flow cytometer (BD Biosciences).

\section{Hoechst 33258 staining}

After treatment with chemotherapeutic agents for $48 \mathrm{~h}$, the Hoechst 33258 staining kit (Life, Eugene, OR, USA) was applied to observe the apoptotic cells. Each assay was carried out at least three times.

\section{RNA pulldown}

RNA pulldown assays were performed to determine the miR-423-5p and GREM2 interaction as previously described $^{21}$.

\section{Luciferase reporter gene assay}

The reverse complementary sequence of miR-423-5p was synthesized and cloned to construct psiCHECK2-423. Likewise, the 3'-UTR of GREM2 containing the miR-423$5 \mathrm{p}$ binding site was amplified and cloned to construct psiCHECK2-GREM2. Then, the psiCHECK2 vector containing a second reporter gene (firefly luciferase) was utilized for endpoint lytic assays. Lipofectamine 2000 (Life Technologies) was applied for reporter transfection. After $48 \mathrm{~h}$, luciferase activity was measured.

\section{Xenograft tumors in nude mice}

BALB/c-nu mice (5 weeks) were randomly assigned to 4 groups $(n=6)$. Parental $22 \mathrm{Rv}-1$ and $22 \mathrm{Rv}-1 / \mathrm{TAX}$ cells $\left(1 \times 10^{7}\right.$ cells $/ 0.1 \mathrm{~mL}$ of PBS) were injected subcutaneously into the left and right flanks of nude mice. One week later, taxane was injected around the tumors on the $12^{\text {th }}, 15^{\text {th }}$, 
a
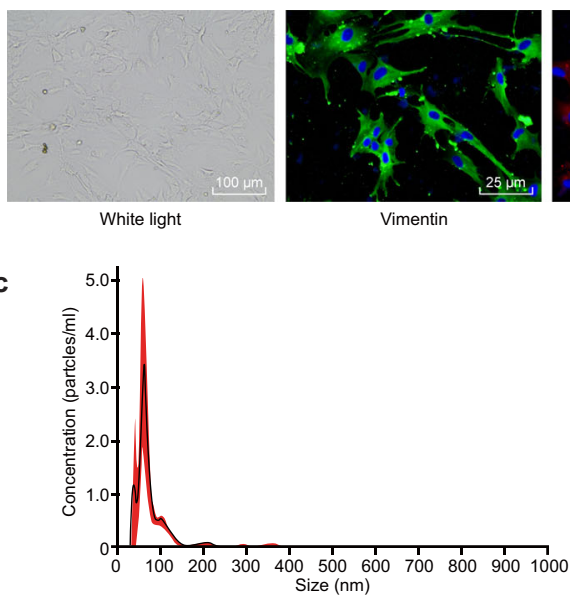

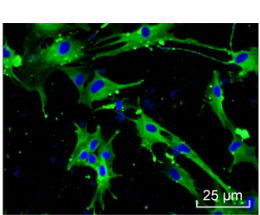

Vimentin Size $(\mathrm{nm})$

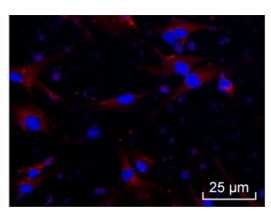

FAP
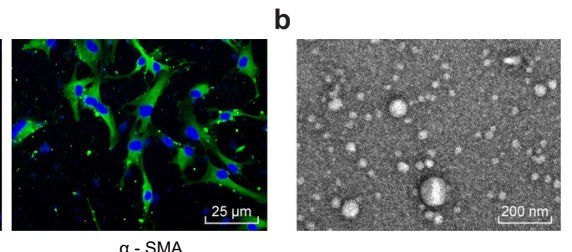

d

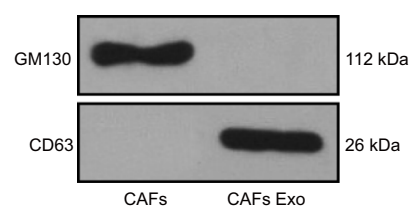

Fig. 1 Characteristics of cancer-associated fibroblasts and isolation of exosomes. a Representative morphology of CAFs (scale bar, 200 um) and immunofluorescence staining identification of CAFs using antibodies against Vimentin, a-SMA, and FAP (scale bar, 100 um). b Transmission electron microscopy showing exosomes isolated from the CAFs (scale bar, $100 \mathrm{~nm}$ ). c Nanoparticle tracking analysis of the CAF-derived exosomes (represented as size vs. concentration). d Western blot analysis of the exosome marker CD63 and the Golgi matrix protein GM130 in exosomeenriched conditioned medium.

and $18^{\text {th }}$ days. When the tumors were visible, the following treatments were performed: (1) inhibitor control and (2) miR-423-5p inhibitor in parental $22 \mathrm{Rv}-1$ and $22 \mathrm{Rv}-1 / \mathrm{TAX}$ subcutaneous mice. The mice were sacrificed on the 35th day, and the tumor volume and weight were measured.

\section{Immunohistochemistry}

Serial Section $(4 \mu \mathrm{m})$ were made for immunohistochemistry for GREM2 (ab228736, Abcam) and Ki67 (ab15580, Abcam) ${ }^{22}$. Five regions were randomly selected to observe the area and the intensity of positive staining by ImagePro Plus 5.1 software, and the average value was obtained to assess the protein levels.

\section{Statistical analysis}

All experiments were performed 3 times. Data are presented as the mean \pm standard deviation (SD). Statistical analysis was performed with GraphPad Prism 8 software. The $p$-values were obtained using an unpaired $t$ test, one-way or two-way analysis of variance (ANOVA), followed by Sidak's multiple comparison test or Tukey's multiple comparison test. $P<0.05$ was regarded as statistically significant.

\section{Results}

Identification of CAFs and CAF-derived exosomes

We first observed that the cultured cells had a long spindle shape, multilayered growth and a disordered arrangement at a certain density, which is a typical cell morphology of CAFs. Then, we identified the CAF surface markers $\alpha$-SMA and FAP and the fibroblast marker
Vimentin by immunofluorescence. $\alpha$-SMA, FAP and Vimentin were positive in the isolated and cultured cells, suggesting the successful isolation of CAFs (Fig. 1a). Subsequently, to investigate the functions of the CAFderived exosomes, we isolated exosomes from the CAFs, and the CAF-derived exosomes were identified by transmission electron microscopy, nanoparticle analysis and Western blot analysis. The transmission electron microscopy results showed that the exosomes were round or oval membrane vesicles with basically the same shape (Fig. 1b). From the results of nanoparticle analysis, we observed that the diameter of the exosomes was approximately $90 \mathrm{~nm}$, and most exosomes had a diameter of 30-150 nm (Fig. 1c). Western blot analysis revealed that CAF-derived exosomes were positive for CD63 but negative for GM130, indicating the successful isolation of CAF-derived exosomes (Fig. 1d).

\section{CAF-derived exosomes promoted drug resistance in PC cells}

To explore the effect of CAF-derived exosomes on drug resistance in PC cells, we first verified the successful construction of LN-CaP/DTX, 22Rv-1/TAX- and C4-2/ BCT-resistant cell lines by CCK- 8 assays $(P<0.05$, Fig. 2a). Subsequently, the exosomes derived from the CAFs were added to the parent LN-CaP, 22Rv-1 and C4-2 cells and the corresponding drug-resistant cells at a dose of $20 \mu \mathrm{g} / \mathrm{mL}$ for culture for $24 \mathrm{~h}$, and then, the cells were treated with $2 \mu \mathrm{M}$ docetaxel, $5 \mu \mathrm{M}$ taxane and $10 \mu \mathrm{M}$ bicalutamide for $2 \mathrm{~h}$. Next, CCK- 8 assays, colony formation assays and flow cytometry were carried out and indicated that compared with the parental cells without 
a

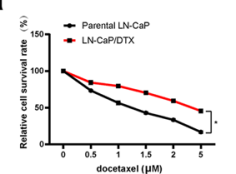

C

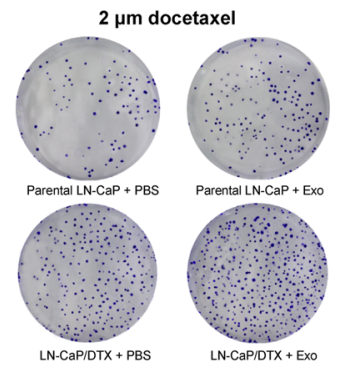

- Parental LN-CaP + PBS

- Parental LN-CaP + Exo

- LN-CaPIDTX +PBS

$\overbrace{60} 7$
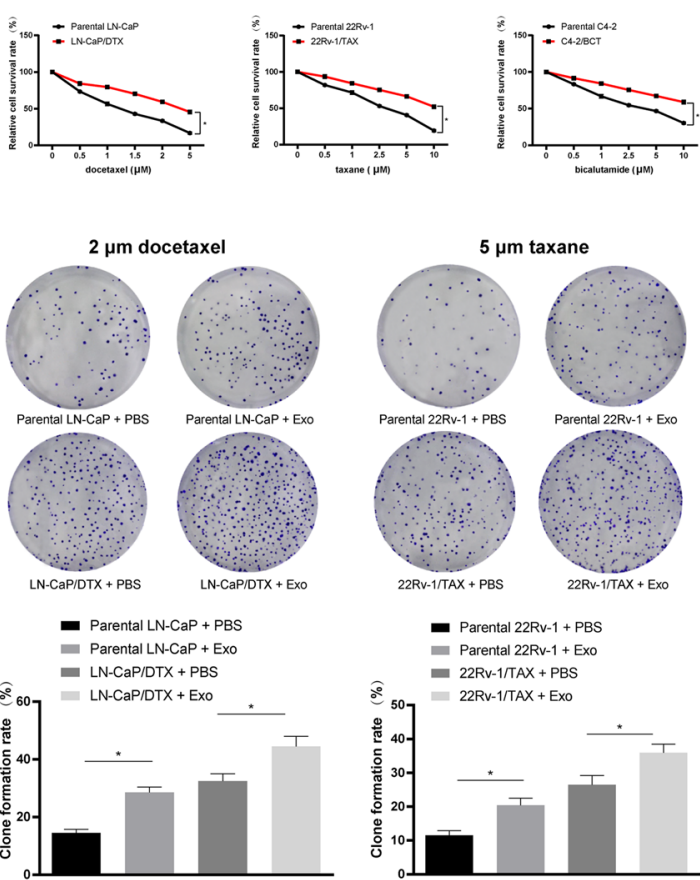

- Parental 22Rv-1 + PBS - Parental 22Rv-1 + Exo

- 22Rv-1/TAX + PBS

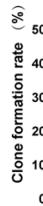
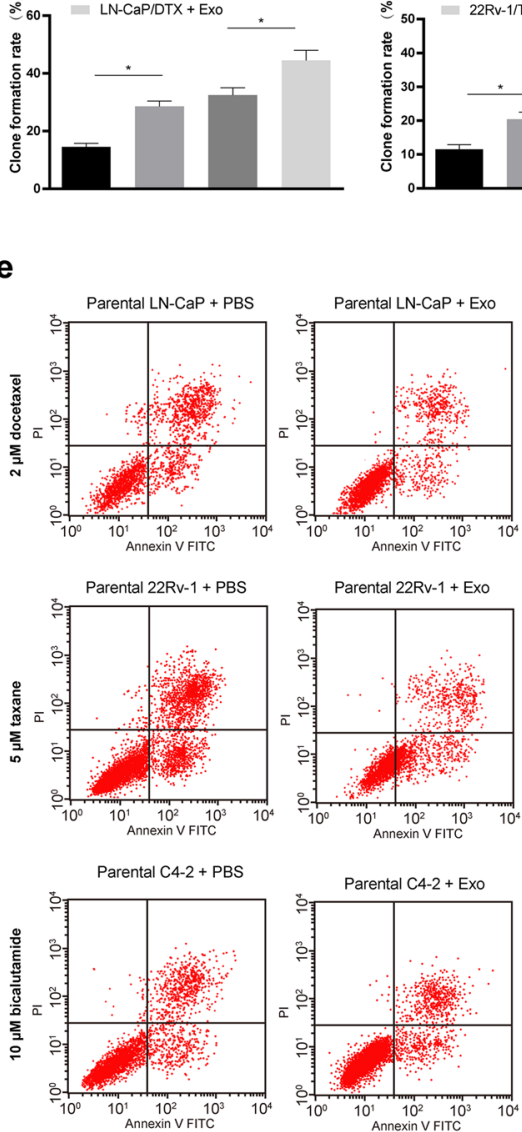
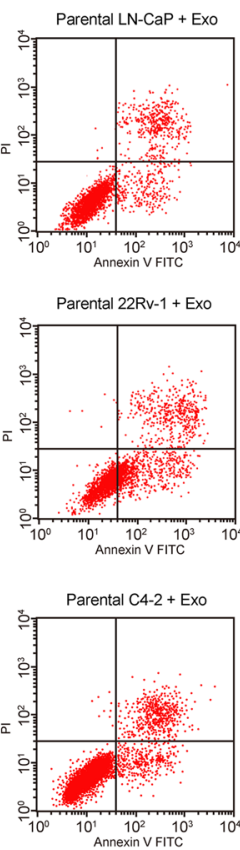
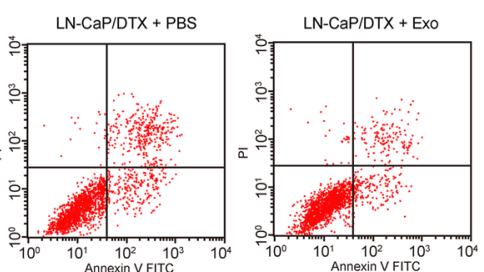
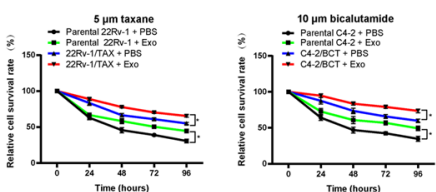

d

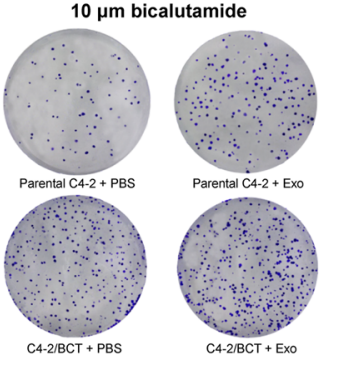

- Parental C4-2 + PBS

- C4-2/BCT + PBS

$\stackrel{Ð}{\circ}^{50} 7$

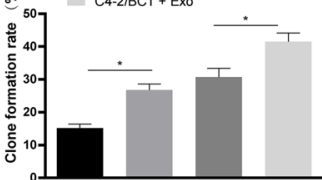

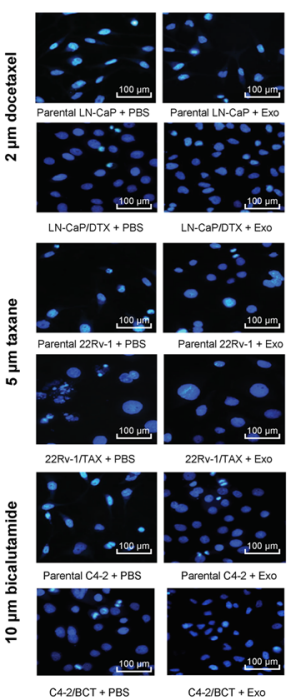

$C 42 / B C T+E \times 0$
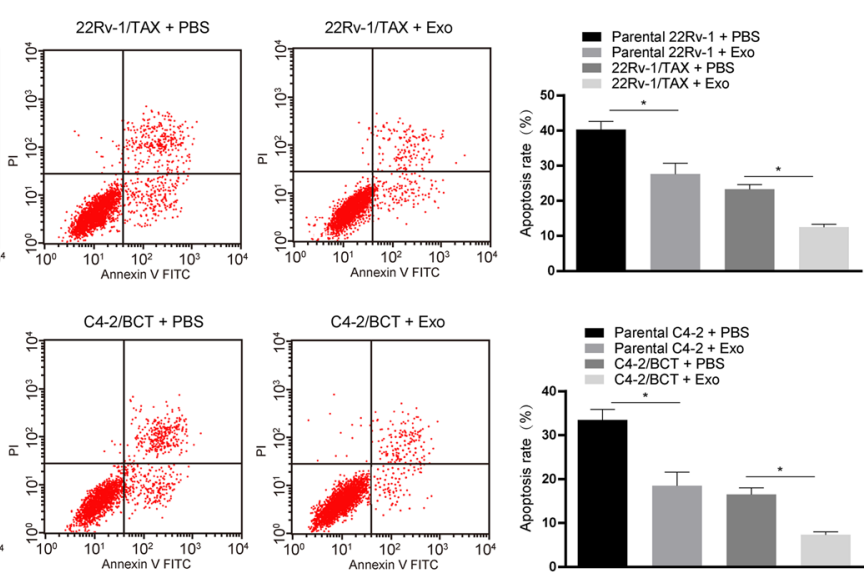

Fig. 2 CAF-derived exosomes promoted drug resistance of prostate cancer cells. a Docetaxel-resistant LN-CaP (LN-CaP/DTX) cells, taxaneresistant 22Rv-1 (22Rv-1/TAX) cells and bicalutamide-resistant C4-2 (C4-2/BCT) cells were exposed to gradient concentrations of drugs (Sigma-Aldrich, St Louis, MO, USA) to generate drug-resistant prostate cancer cells, as shown by CCK-8 assays. Then, the parental or drug-resistant prostate cancer cells were treated with $20 \mu \mathrm{g} / \mathrm{mL}$ CAF-derived exosomes (Exo group) or PBS as a negative control (PBS group) for $24 \mathrm{~h}$. Continuously, CAF-derived exosome-treated parental or drug-resistant prostate cancer cells were exposed to $2 \mu \mathrm{M}$ docetaxel, $5 \mu \mathrm{M}$ taxane and $10 \mu \mathrm{M}$ bicalutamide for $2 \mathrm{~h}$. Then, CCK-8 assays (b), colony formation assays (c), Hoechst 33258 staining (d) and flow cytometry (e) were performed to determine the effectiveness of the CAF-derived exosomes. Data are expressed as the mean \pm standard deviation. One-way ANOVA and Sidak's multiple comparisons test were used to determine statistical significance or two-way ANOVA and Tukey's multiple comparisons test were used, ${ }^{*} P<0.05$. Three independent experiments were performed. 
a

b

C
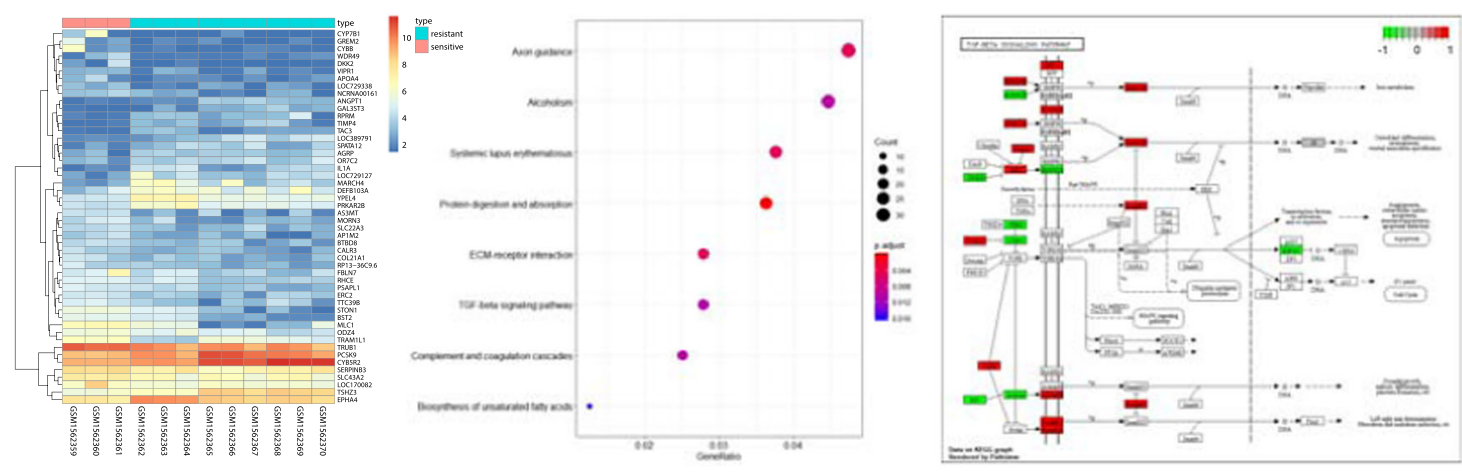

d

e
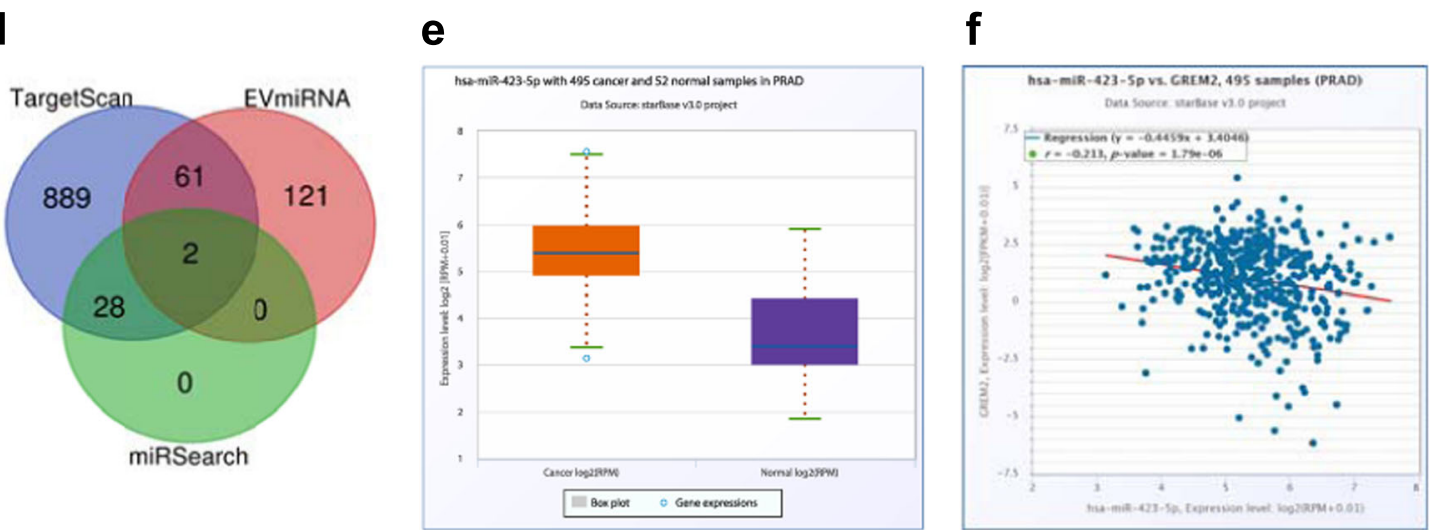

\section{g}

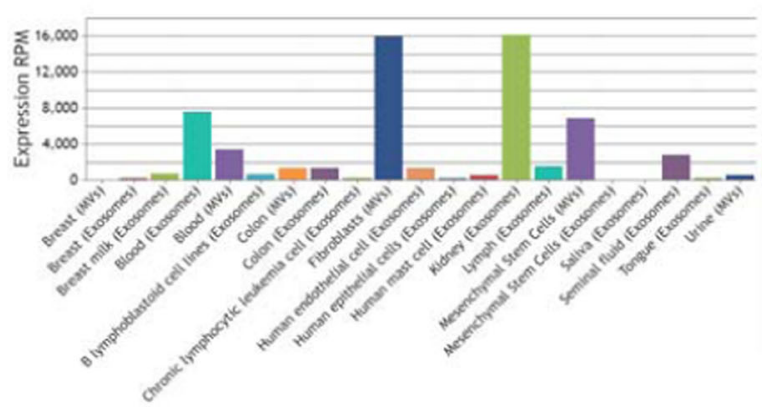

Parental LN-CaP + PBS
Parental LN-CaP + Exo

LN-CaP/DTX + PBS

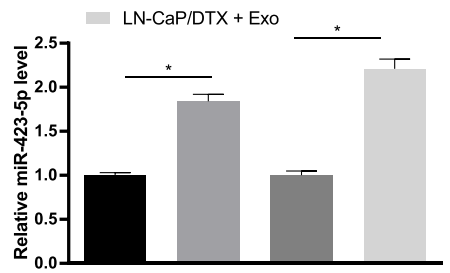

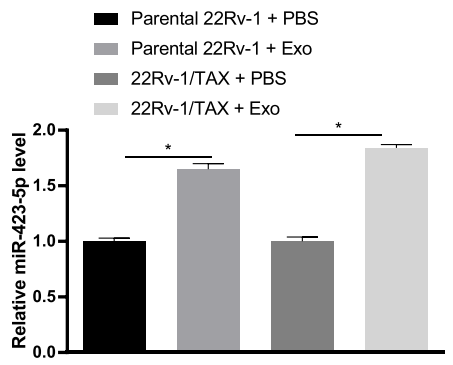

h
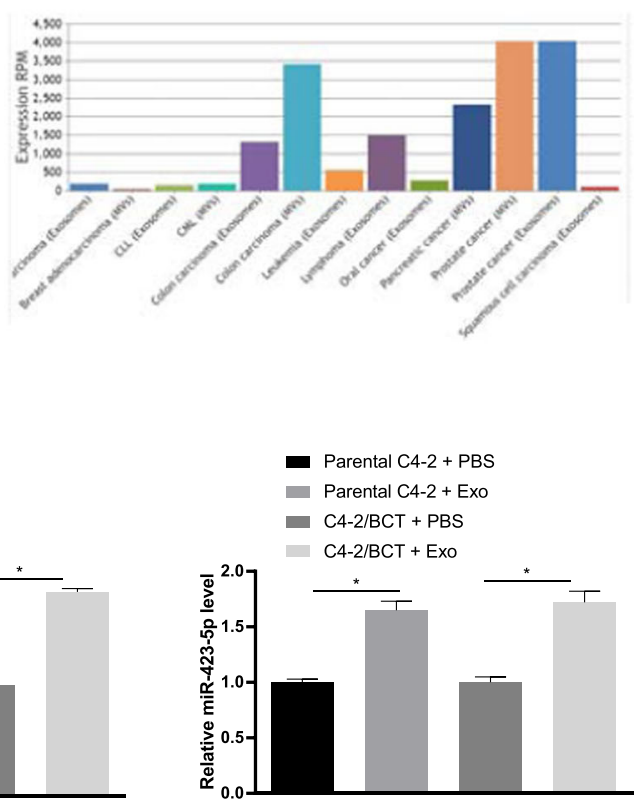

Fig. 3 (See legend on next page.) 
(see figure on previous page)

Fig. 3 CAF-secreted exosomal miR-423-5p promoted drug resistance in prostate cancer. a The prostate cancer resistance chip GSE64012 was used; transverse coordinates represent the sample number, longitudinal coordinates represent the gene names, the left dendrogram shows gene expression clustering, and the upper right histogram shows the color gradation. $\mathbf{b}$ Enrichment analysis of KEGG pathways. The horizontal coordinates represent the GeneRatio, the vertical coordinates show the KEGG entry, and the right histogram is the color gradation. c Position and differential expression of differentially expressed genes in the TGF- $\beta$ signaling pathway; red indicates genes that were highly expressed in the drug-resistant samples, and green indicates genes that were lowly expressed in the drug-resistant samples. $\mathbf{d}$ Prediction of miRNAs regulated upstream of GREM2. The three circles in the figure represent the prediction results of the two databases, and the miRNAs, the intermediate part of the EVmiRNA database, which were highly expressed in the fibroblasts microbubbles, represent the intersection of the three groups of data. e Expression of miR-423-5p in prostate cancer samples from TCGA. Horizontal coordinates represent the sample types, and vertical coordinates represent expression. $\mathbf{f}$ Correlation analysis of miR-423-5p and GREM2 expression in prostate cancer samples from TCGA. $\mathbf{g}, \mathbf{h}$ Expression of miR-423-5 $p$ in exosomes from different samples and exosomes from different tumors. Horizontal coordinates represent sample types, and vertical coordinates represent miRNA expression. $\mathbf{i}$ RT-qPCR was used to detect the expression of miR-423-5p in the prostate cancer cells treated with CAF-derived exosome, with normalization to U6. Data are expressed as the mean \pm standard deviation. One-way ANOVA and Sidak's multiple comparisons test were used to determine statistical significance or two-way ANOVA and Tukey's multiple comparisons test were used, ${ }^{*} P<0.05$. Three independent experiments were performed.

treatment, the parental cells treated with the CAF-derived exosomes showed enhanced proliferation and colony formation and a decreased apoptosis rate. Compared with the drug-resistant cells without treatment, the drugresistant cells treated with the CAF-derived exosomes showed enhanced proliferation and colony formation and a decreased apoptosis rate $(P<0.05$, Fig. $2 \mathrm{~b}-\mathrm{e})$. These results demonstrated that the CAF-derived exosomes decreased the chemosensitivity of the parental PC cells and enhanced the drug resistance of the drug-resistant cells.

\section{CAF-secreted exosomal miR-423-5p promoted drug resistance in $\mathrm{PC}$}

The PC drug resistance chip GSE64012 was obtained from the GEO database. The chip includes 3 taxanesensitive samples and 9 taxane-resistant samples. Using sensitive samples as a control, we used the $\mathrm{R}$ language "limma" package to analyze the differences, and 1964 significant differentially expressed genes in the drug resistance samples were obtained with $|\operatorname{logFC}|>2$ and $P<$ 0.05 as the screening criteria. Figure $3 \mathrm{a}$ shows the expression heat map of some of the significantly differentially expressed genes. To further elucidate the drugrelated signaling pathways, we analyzed these differentially expressed genes by KEGG pathway enrichment analysis, and the results showed that some differentially expressed genes were enriched in the TGF- $\beta$ pathway (Fig. 3b). The TGF- $\beta$ pathway is an important regulatory pathway in tumor development ${ }^{23,24}$. In the TGF- $\beta$ pathway, 20 differentially expressed genes were enriched (Fig. $3 c)$. We noted that the GREM2 gene was upstream of the TGF- $\beta$ pathway, and its expression in the drug-resistant PC samples was significantly decreased, which indicated that it likely affected the resistance of PC to taxane chemotherapy by regulating TGF- $\beta$. Tumor-related fibroblasts influence the resistance of tumor cells to chemotherapy ${ }^{25}$. To further elucidate the upstream regulatory mechanism of GREM2, we predicted the upstream
miRs of GREM2 using the TargetScan database and the miRSearch database. Furthermore, highly expressed miRs in fibroblast microvesicles were obtained from the EVmiRNA database. After determining the intersection of the predicted results and the highly expressed miRs in fibroblast microvesicles, we found that miR-423-5p and miR-382-3p were located in the intersection, and miR423-5p showed a higher average expression value in fibroblast microvesicles than miR-382-3p (Fig. 3d). The average miR-423-5p expression was higher in the PC samples than in the normal samples, as shown by TCGA analysis (Fig. 3e). Correlation analysis was performed on the expression of miR-423-5p and GREM2 in TCGA, and we found that miR-423-5p was negatively correlated with the expression of GREM2 (Fig. 3f). In addition, miR-423$5 \mathrm{p}$ was highly expressed in fibroblast microvesicles and PC exosomes (Fig. 3g, h). Furthermore, miR-423-5p expression increased significantly in the PC cells after treatment with the CAF-derived exosomes $(P<0.05$, Fig. 3i). These results suggested that the PC-associated fibroblast-derived exosomes carrying miR-423-5p increased the resistance of PC to taxane by inhibiting GREM2 through the TGF- $\beta$ pathway.

\section{Inhibition of miR-423-5p attenuated the drug resistance of $\mathrm{PC}$ cells via CAF-derived exosome treatment}

To further evaluate the effect of CAF-secreted exosomal miR-423-5p on the drug resistance of PC cells, we inhibited miR-423-5p expression in CAFs through transfection of a miR-423-5p inhibitor (transfection of an inhibitor control was regarded as a control) and then extracted the exosomes. After miR-423-5p intervention, miR-423-5p expression in the CAFs and the CAF-Exos decreased significantly, as determined by RT-qPCR $(P<$ 0.05, Fig. 4a). In addition, we treated the parental cells and the drug-resistant cells with the extracted exosomes (after transfection of the miR-423-5p inhibitor in the CAFs) (Exo/inhibitor control and Exo/miR-423-5p inhibitor). Then, CCK- 8 assays, colony formation assays and flow 
a

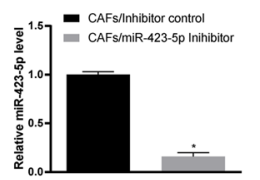

C
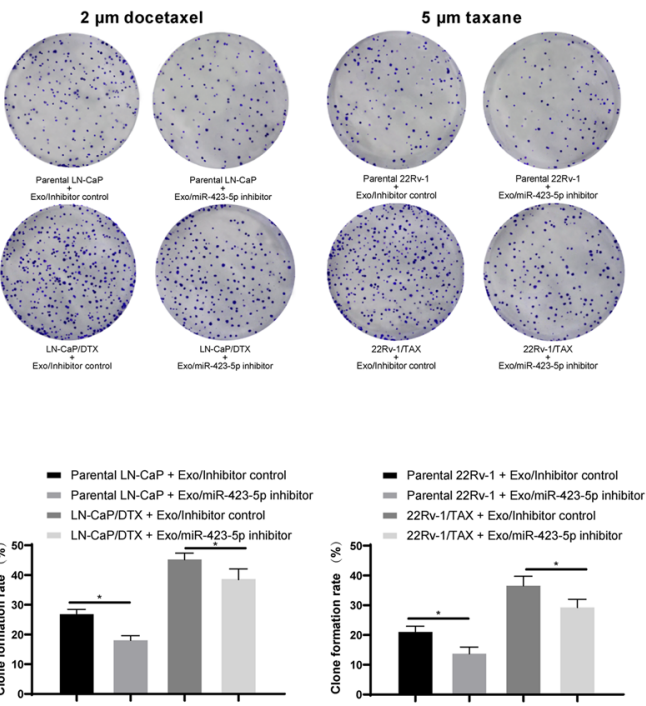

e

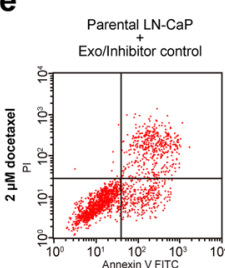

Parental 22Rv-1

Exo/lnhibitor contro

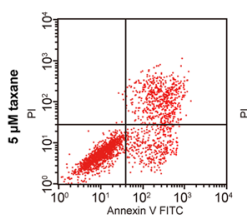

Parental C4-2 Exolnhibitor contro
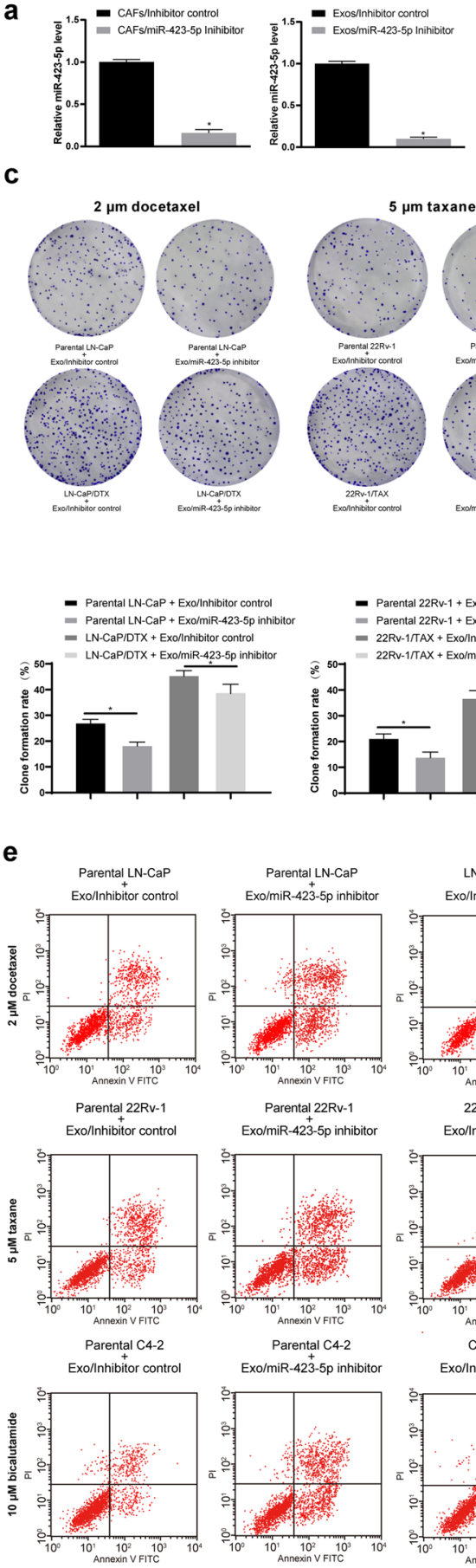
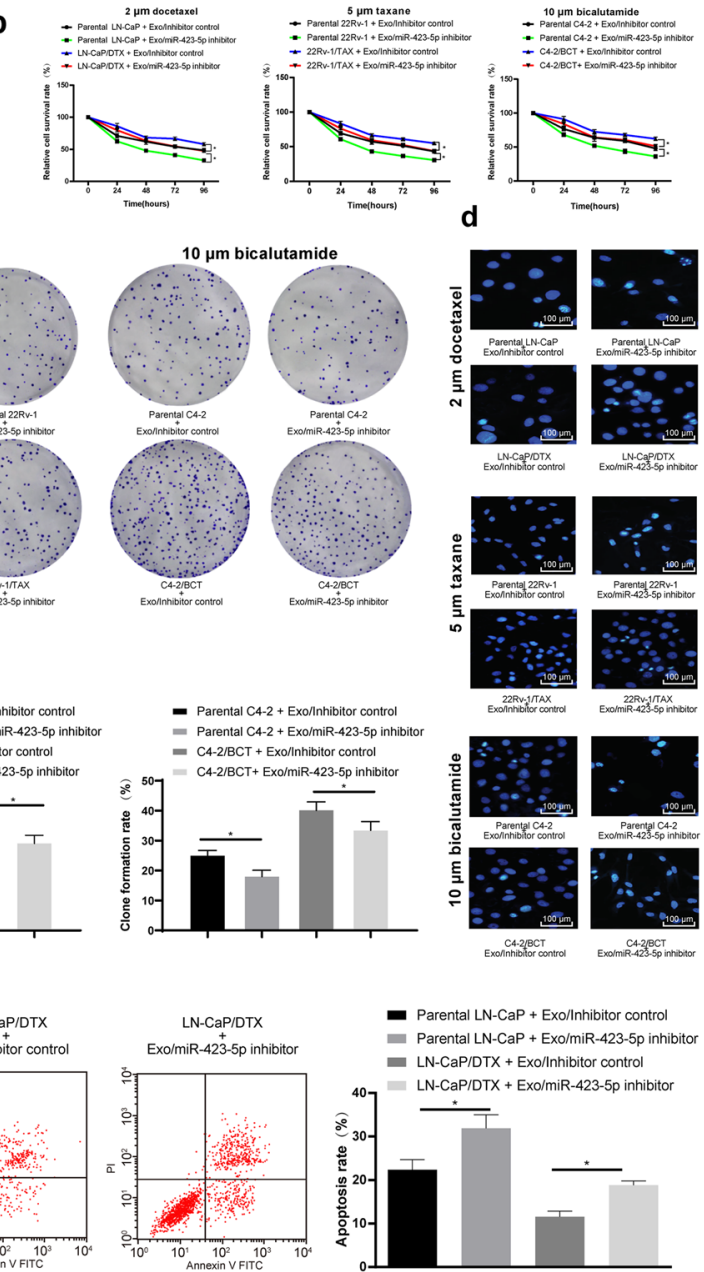

$22 R v-1 / T A X$

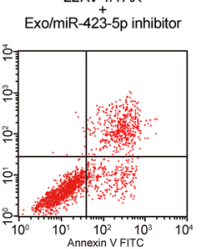

C4-2/BCT
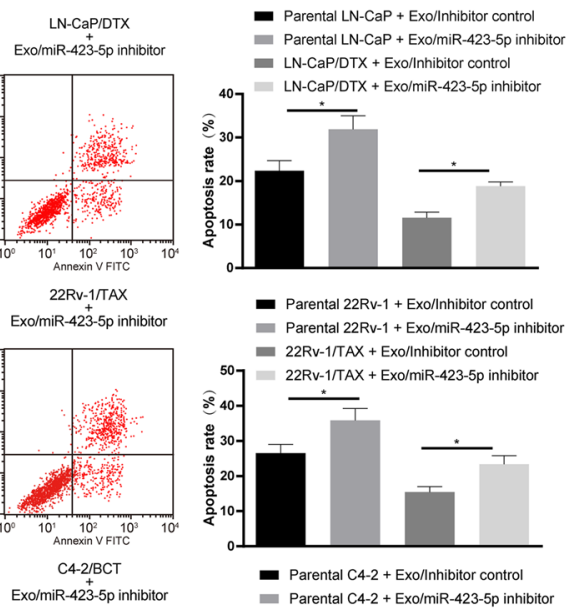

- Parental 22Rv-1 + Exo/lnhibitor control

- Parental 22Rv-1 + Exo/miR-423-5p inhibitor

- 22Rv-1/TAX + Exo/lnhibitor control

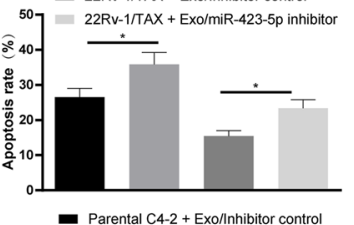

tal C4-2 + Exo/nhibitor control

- Parental C4-2 + Exo/mir-423-5p in

- $C 4-2 / B C T+$ Exo/lnhibitor control

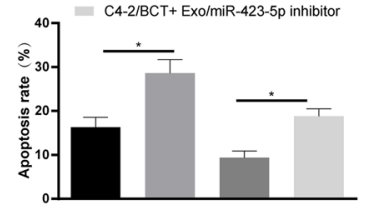

Fig. 4 Inhibition of miR-423-5p attenuated the drug resistance of prostate cancer cells via CAF-derived exosome treatment. The miR-423-5p inhibitor was transfected into CAFs, while the inhibitor control served as the negative control. Then, exosomes (Exo/inhibitor control group and Exo/ miR-423-5p inhibitor group) were extracted as described in the "Materials and methods" section. a RT-qPCR was performed to determine miR-423-5p expression in the CAFs and the exosomes. Continuously, CAF-derived exosome-treated parental or drug-resistant prostate cancer cells were exposed to $2 \mu \mathrm{M}$ docetaxel, $5 \mu \mathrm{M}$ taxane and $10 \mu \mathrm{M}$ bicalutamide for $2 \mathrm{~h}$. Then, CCK-8 assays (b), colony formation assays (c), Hoechst 33258 staining (d) and flow cytometry analysis (e) were performed to determine the effectiveness of the CAF-derived exosomes. Data are expressed as the mean \pm standard deviation. One-way ANOVA and Sidak's multiple comparisons test were used to determine statistical significance or two-way ANOVA and Tukey's multiple comparisons test were used, ${ }^{*} P<0.05$. Three independent experiments were performed. 


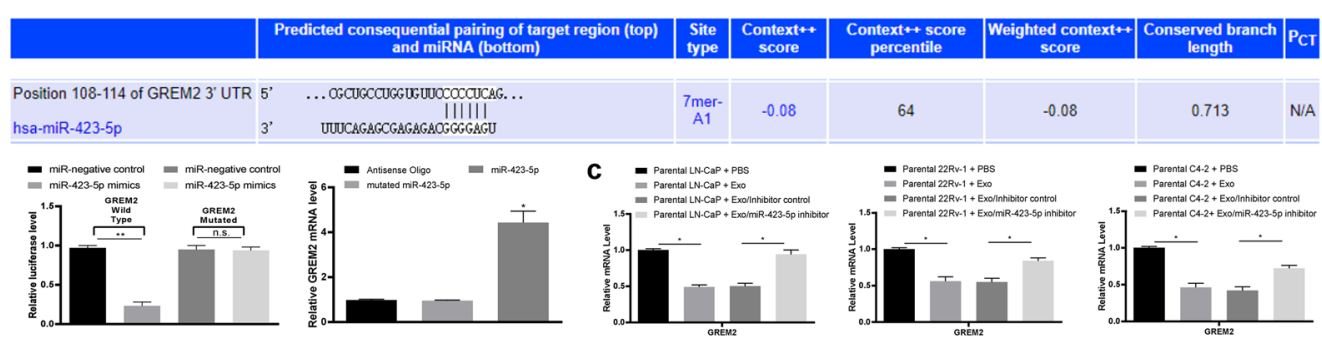

d
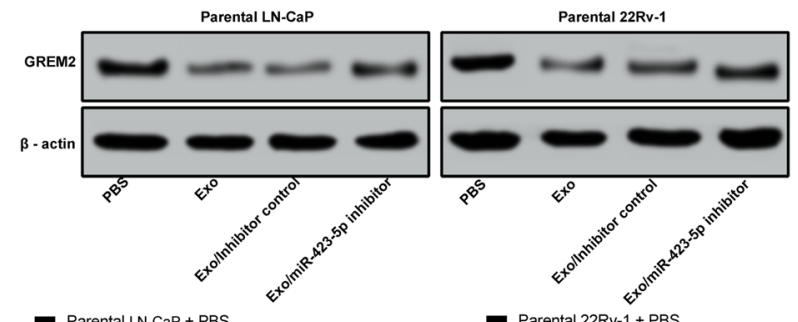

- Parental LN-CaP + PBS

- Parental 22Rv-1 + PBS

- Parental LN-CaP + Exo/lnhibitor control

- Parental 22Rv-1 + Exo

- Parental 22Rv-1 + Exo/Inhibitor control

- Parental LN-CaP + Exo/miR-423-5p inhibitor
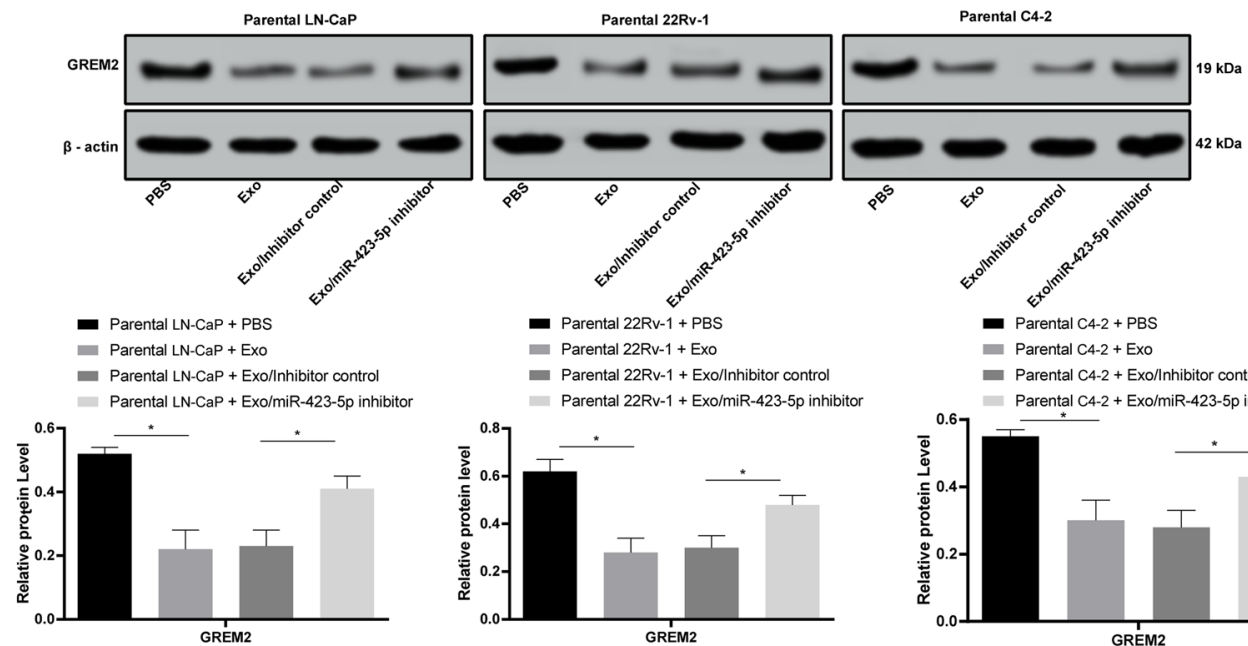

- Parental C4-2 + PBS

- Parental C4-2 + Exo

- Parental $C 4-2+$ Exo/lnhibitor control

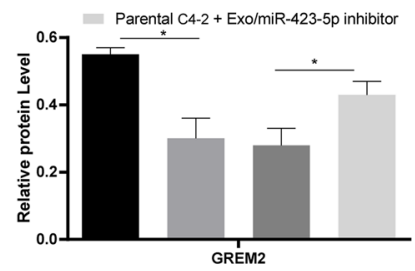

e
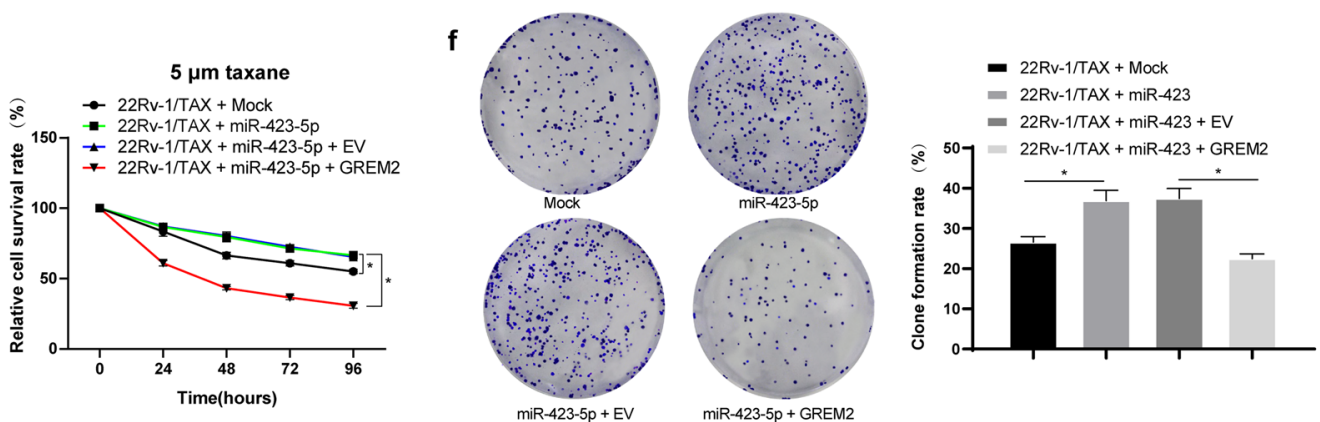

g
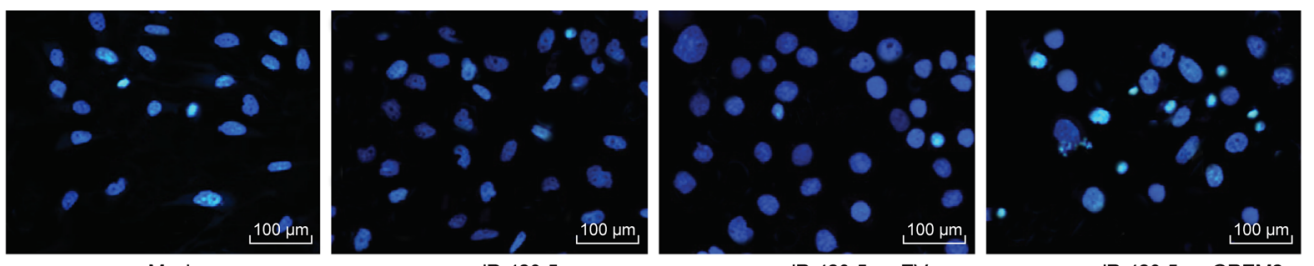

h

- 22Rv-1/TAX + Mock

= 22Rv-1 $1 \mathrm{TAX}+$ miR-423 + EV
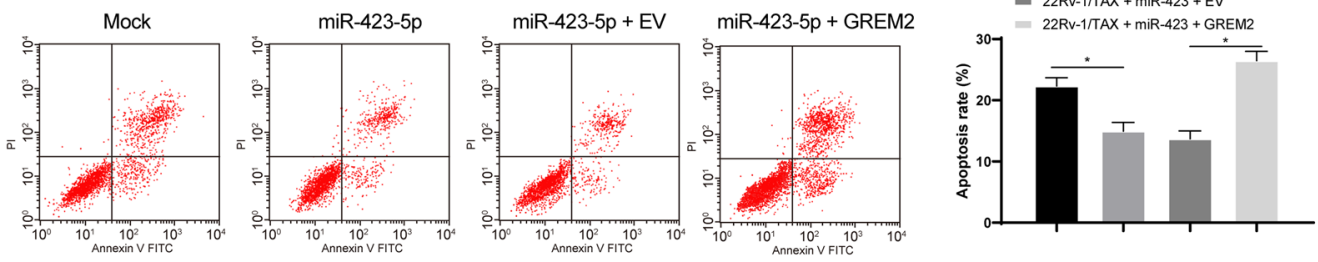

Fig. 5 (See legend on next page.) 
(see figure on previous page)

Fig. 5 CAF-secreted exosomal miR-423-5p promoted chemotherapy resistance of prostate cancer by targeting GREM2. a TargetScan predicted that miR-423-5p could bind to GREM2 sequences. b Luciferase reporter plasmid containing GREM2-WT or GREM2-Mut was transfected into 293T cells together with miR-423-5p in parallel with a miR-NC plasmid vector. The enrichment of miR-423-5p on GREM2 was detected by RNA pulldown-qPCR assays relative to antisense oligos. Furthermore, RT-qPCR and Western blot analysis were performed to validate the GREM2 mRNA (c) and protein (d) levels. miR-423-5p was transfected into 22Rv-1/TAX cells, and a GREM2 expression vector was transfected. The miR-negative control (mock group) and empty vector (EV group) served as the negative control. Cells were exposed to $5 \mu \mathrm{M}$ taxane for $2 \mathrm{~h}$. Then, CCK-8 assays (e), colony formation assays $(\mathbf{f})$, Hoechst 33258 staining $(\mathbf{g})$ and flow cytometry analysis $(\mathbf{h})$ were performed. The expression of miR-423-5p was normalized to that of U6, while the GREM2 mRNA level was normalized to that of GAPDH. Data are expressed as the mean \pm standard deviation. One-way ANOVA and Sidak's multiple comparison test were used to determine statistical significance or two-way ANOVA and Tukey's multiple comparison test were used, ${ }^{*} P<0.05,{ }^{*} P<0.01$. Three independent experiments were performed.

cytometry were carried out and indicated that compared with the Exo/inhibitor control treatment, the Exo/miR423-5p inhibitor treatment could significantly inhibit proliferation and colony formation of the parental cells and the drug-resistant cells while increasing the apoptosis rate, suggesting that the decrease in exosomal miR-423-5p partially reversed the decrease in drug sensitivity and the increase in resistance of PC cells induced by CAF-Exo treatment $(P<0.05$, Fig. $4 \mathrm{~b}-\mathrm{e})$.

\section{CAF-secreted exosomal miR-423-5p promoted chemotherapy resistance of PC by targeting GREM2}

To further explore the downstream mechanism of miR423-5p, we designed a double-luciferase experiment based on the binding sequence of miR-423-5p and GREM2 in TargetScan, and the results confirmed that miR-423-5p could target GREM2. Then, RNA pulldown experiments verified that miR-423-5p could target the 3'-UTR sequence of GREM2 $(P<0.05$, Fig. $5 \mathrm{a}$, b). Subsequently, to verify the effect of miR-423-5p on GRAM2 expression, we determined the GREM2 levels in the PC cells treated with the CAF-derived exosomes, and the results showed that the GREM mRNA and protein levels in the PC cells treated with the CAF-derived exosomes were significantly decreased, while obvious changes were observed in the PC cells after exosomal miR-423-5p was decreased $(P<0.05$, Fig. 5c, d). Additionally, to verify that miR-423-5p carried by the CAF-derived exosomes promoted chemotherapy resistance in prostate cancer by targeting GREM2, we selected 22Rv-1/TAX cells for functional rescue experiments. We overexpressed miR-423-5p in the $22 \mathrm{Rv}-1 / \mathrm{TAX}$ cells and found that the resistance to taxane was increased in the $22 \mathrm{Rv}-1$ cells, as evidenced by the enhanced cell proliferation and colony formation and the decreased cell apoptosis rate. Furthermore, the resistance to taxane in the $22 \mathrm{Rv}-1 / \mathrm{TAX}$ cells decreased significantly after combined overexpression of miR-423-5p and GREM2 compared with overexpression of miR-423-5p only, as evidenced by the inhibited cell proliferation and colony formation and the increased cell apoptosis rate $(P<0.05$, Fig. 5e-h).
Inhibition of TGF- $\beta$ pathway activity partially reversed the increase in drug resistance in PC cells induced by the CAFderived exosomes

According to the results of Fig. 3, the TGF- $\beta$ mRNA and protein expression in $\mathrm{PC}$ cells was detected. Exosome treatment increased the TGF- $\beta$ mRNA and protein expression in the PC cells, while the TGF- $\beta$ mRNA and protein expression in the $\mathrm{PC}$ cells increased after reducing exosomal miR-423-5p $(P<0.05$, Fig. 6a, b). The above results further demonstrated that CAF-secreted exosomal miR-423-5p promoted the TGF- $\beta$ pathway by targeting GREM2.

To further determine the role of the TGF- $\beta$ pathway in the taxane resistance of $\mathrm{PC}$ cells after treatment with the CAF-derived exosomes, we selected $22 \mathrm{Rv}-1$ and $22 \mathrm{Rv}-1 /$ TAX cells for functional rescue experiments, and the TGF- $\beta$-specific inhibitor LY2109761 was used to treat the $22 \mathrm{Rv}-1$ cells pretreated with the CAF-derived exosomes. Then, CCK- 8 assays, colony formation assays and flow cytometry were carried out, and the results showed that TGF- $\beta$ inhibition could partially reverse the increase in cell proliferation and colony formation as well as the decrease in the cell apoptosis rate, suggesting that TGF- $\beta$ inhibition could suppress taxane resistance in the $22 \mathrm{Rv}-1$ cells induced by the CAF-derived exosomes $(P<0.05$, Fig. 6c-g).

\section{miR-423-5p inhibition increased the drug sensitivity of the $P C$ cells in vivo}

The effect of miR-423-5p on the growth of the $22 \mathrm{Rv}-1$ parental cells and the drug-resistant cells in vivo was evaluated by measuring the growth and weight of transplanted tumors in nude mice. The results showed that compared with the inhibitor control treatment, the miR423-5p inhibitor treatment prevented tumor growth and reduced taxane resistance $(P<0.05$, Fig. $7 \mathrm{a})$ and decreased the tumor weight $(P<0.05$, Fig. $7 \mathrm{~b})$. The positive expression of the proliferation marker Ki-67 detected by immunohistochemistry was significantly reduced $(P<$ 0.05 , Fig. $7 c$ ), indicating that inhibition of miR-423-5p expression could reduce the taxane resistance of PC cells 


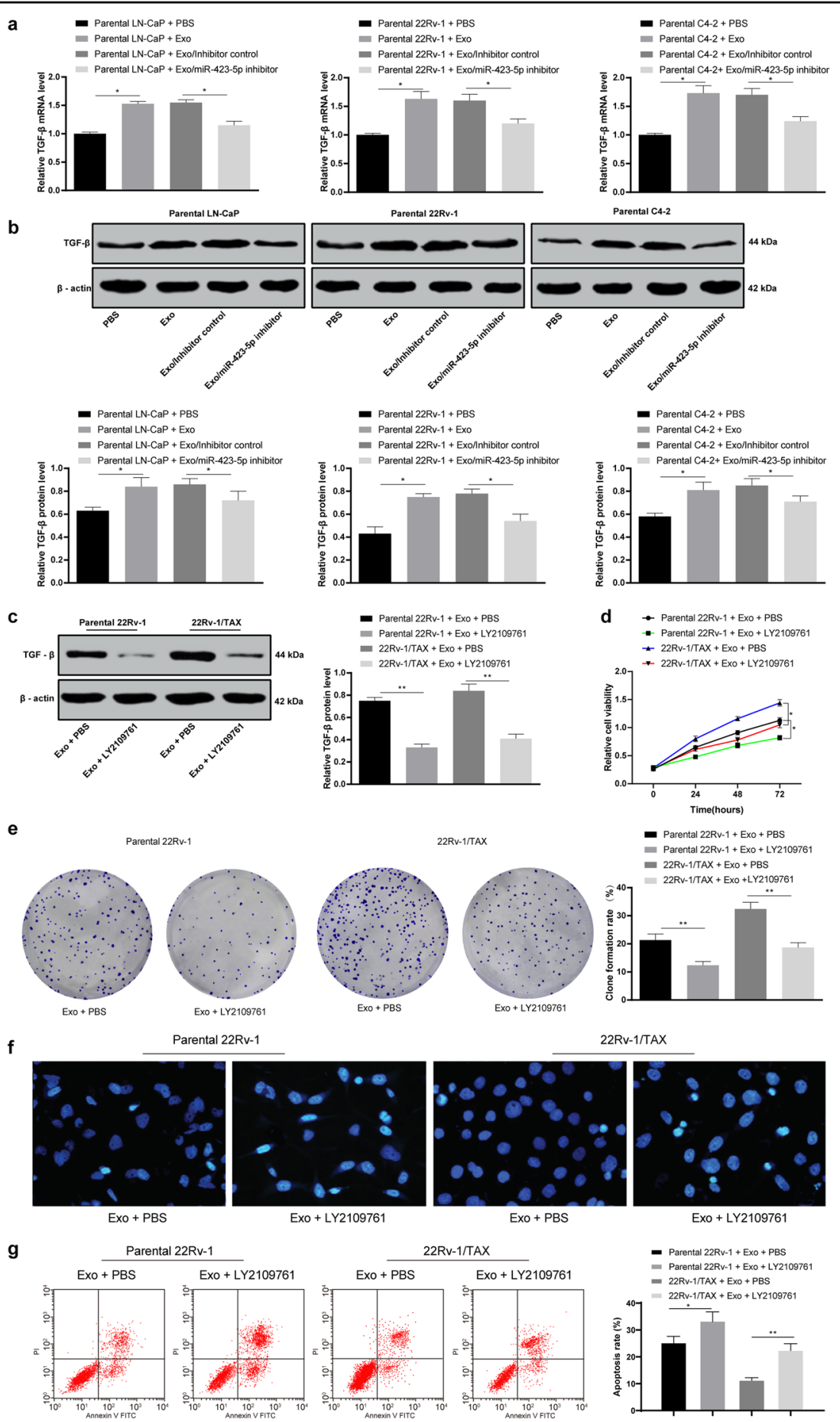

Fig. 6 LY2109761 treatment partially reversed taxane resistance in prostate cancer cells via CAF-derived exosome treatment. RT-qPCR and Western blot analysis were performed to validate the TGF- $\beta$ mRNA (a) and protein levels (b). LY2109761 is a TGF- $\beta$-specific inhibitor, and $15 \mu M$ LY2109761 was added to parental or taxane-resistant 22Rv-1 cells cocultured with the CAF-derived exosomes. Western blot analysis was performed to determine the TGF- $\beta$ protein levels (c). Then, the cells were exposed to $5 \mu \mathrm{M}$ taxane for $2 \mathrm{~h}$. Then, CCK-8 assays (d), colony formation assays (e), Hoechst 33258 staining $(\mathbf{f})$ and flow cytometry analysis $(\mathbf{g})$ were performed to determine the effectiveness of LY2109761. Data are expressed as the mean \pm standard deviation. One-way ANOVA and Sidak's multiple comparison test were used to determine statistical significance or two-way ANOVA and Tukey's multiple comparisons test were used, ${ }^{*} P<0.05,{ }^{*} P<0.01$. Three independent experiments were performed. 


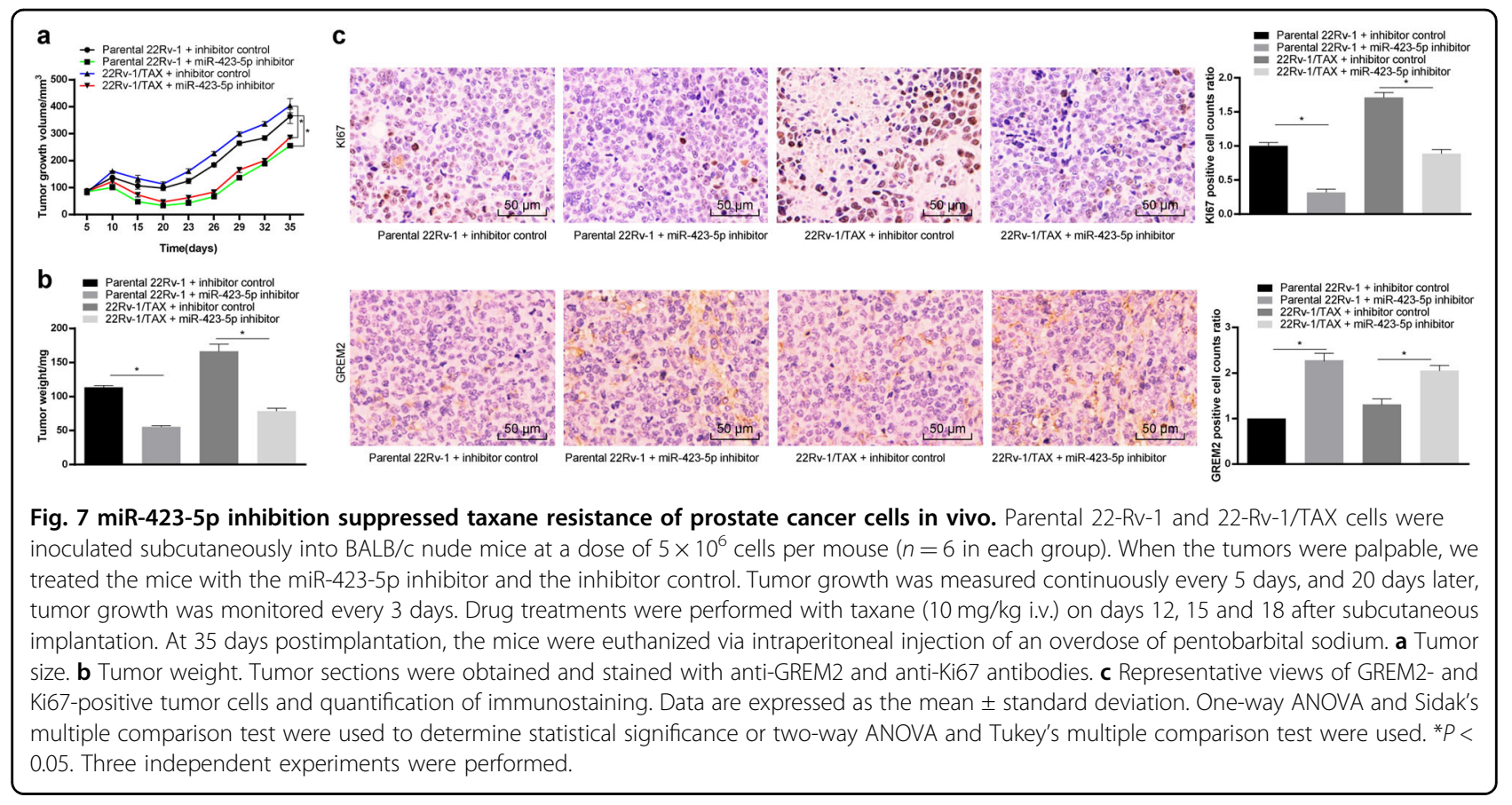

in vivo. In addition, the results of GREM2 immunohistochemistry showed that the GREM2-positive cells increased after inhibiting miR-423-5p expression $(P<$ 0.05 , Fig. 7c). The targeted inhibitory effect of miR-423-5p on GREM2 was confirmed in vivo.

\section{Discussion}

PC is the most common lethal malignancy ${ }^{26}$. miRNAs transferred by exosomes play important roles in the tumor microenvironment ${ }^{27,28}$. In the present study, we explored exosomal communication within the PC microenvironment. Our research demonstrated that miR423-5p was upregulated in the CAF-derived exosomes and that miR-423-5p could promote chemotherapy resistance in PC by targeting GREM2 through the TGF- $\beta$ pathway. These findings indicated that miR-423-5pdevoid exosomes derived from CAFs contribute to the malignant progression of PC.

Initially, our results revealed that GREM2 was substantially decreased in the drug-resistant PC samples, while miR-423-5p expression was increased in the PC samples. As shown in a previous study, the abnormal expression of miRs exerts different effects in multiple kinds of malignancies, including $\mathrm{PC}^{14}$. Previously, miR423-5p expression was found to be upregulated in stable coronary artery disease, heart failure, acute myocardial infarction, and patients undergoing cardiac surgery ${ }^{29,30}$. Lin et al. proved that miR-423-5p was upregulated in human PC tissues and cells ${ }^{31}$. A previous study confirmed that GREM2 downregulation may be a marker in obese mice and humans ${ }^{32}$. However, no research has focused on its expression in PC. In addition, we found that miR-423-5p could target GREM2.

Our present study also showed that treatment with the CAF-derived exosomes decreased the chemosensitivity of the parental PC cells and enhanced the drug resistance of the drug-resistant cells. Accumulating evidence has shown that CAFs can stimulate invasion in breast cancer and non-small-cell lung cancer ${ }^{33,34}$. Additionally, CAFs were reported to advance tumor progression via specific communication within cancer cells. For example, Jing et al. demonstrated that epithelial ovarian cancer (EOC) cells could activate normal omentum via the TGF- $\beta 1$ pathway and that CAFs could result in EOC cell invasion and adhesion ${ }^{35}$. In cancer cells, chemotherapeutic drugs can be delivered via exosomes ${ }^{36}$. Interestingly, studies have noted that oncogenic exosomes could accelerate invasion and drug resistance by delivering oncogenic DNA to normal cells ${ }^{37-39}$. Partly consistent with our study, previous studies also clarified that exosomes derived from CAFs could increase chemoresistance in colorectal and breast cancer cells ${ }^{37,38}$.

Furthermore, we found that the prostate cancerassociated fibroblast-derived exosomes carrying miR423-5p increased the resistance of PC cells to taxane by inhibiting GREM2 through the TGF- $\beta$ pathway. A recent study indicated that tumor-related fibroblasts can influence the resistance of tumors to chemotherapy ${ }^{25}$. CAFs known as myofibroblasts are induced and regulated by TGF- $\beta^{40-42}$. Exosomes derived from PC cells can provide TGF- $\beta$ to transform fibroblasts to myofibroblasts via activating the TGF- $\beta$ /SMAD3 pathway ${ }^{43,44}$. It has also 
been proven that exosomes containing miRs serve as markers for $\mathrm{PC}^{13}$. Previous data suggested that CAFderived exosomes could deliver miRs to neighboring epithelia, leading to increases in $\mathrm{PC}$ cells ${ }^{45}$. One recent report analyzed 29 paclitaxel-resistant PC cell-derived exosomal miRs and demonstrated that these exosomal miRs may result in PC chemoresistance ${ }^{46}$. Moreover, Lin $\mathrm{H}$ et al. confirmed that miR-423-5p inhibition suppressed PC cell growth and tumor volume ${ }^{31}$. CAFs can produce TGF- $\beta$, which in turn activates oncogenic signaling in tumor epithelial cells through the SMAD group of transcription factors ${ }^{47}$. Thus, we believe that blockade of the TGF- $\beta$ pathway partially reversed the increase in drug resistance in PC cells induced by the CAF-derived exosomes.

In this study, we purified exosomes from CAFs and described their activities in vitro and in vivo. We further examined miR expression in exosomes. More importantly, we demonstrated that miR-423-5p was crucial in the chemoresistance of PC cells. This study indicated that CAF-derived exosomes could potentially be exploited as a therapeutic tool for PC.

\begin{abstract}
Acknowledgements
This work was partially supported by the Natural Science Foundation of Jiangsu Province (Grant no. BK20181133), the Key Project in Natural Science Research in Higher Education Institutions of Anhui Province (Grant no. KJ2019A1096, KJ2018A0814) and the Scientific Research Project of Anhui Provincial Health and Family Planning Commission (Grant no. 2018SEYL008).
\end{abstract}

\section{Author details}

${ }^{1}$ Department of Urology, RenMin Hospital of Wuhan University, Wuhan 430060 Hubei, China. 'Department of Medical Laboratory Science, The Fifth People's Hospital of Wuxi, Nanjing Medical University, Wuxi 214000 Jiangsu, China. ${ }^{3}$ Department of Pathology, The Fifth People's Hospital of Wuxi, The Medical School of Jiangnan University, Wuxi 214000 Jiangsu, China. ${ }^{4}$ Center for Precision Medicine, Anhui No. 2 Provincial People's Hospital, Hefei 230041 Anhui, China. ${ }^{5}$ Department of Biology, College of Arts \& Science, Massachusetts University, Boston 02125 MA, USA. ${ }^{6}$ Department of Oncology, RenMin Hospital of Wuhan University, Wuhan 430060 Hubei, China

\section{Conflict of interest}

The authors declare that they have no conflict of interest.

\section{Publisher's note}

Springer Nature remains neutral with regard to jurisdictional claims in published maps and institutional affiliations.

Received: 7 November 2019 Revised: 6 March 2020 Accepted: 10 March 2020.

Published online: 4 November 2020

\section{References}

1. Ferlay, J. et al. Cancer incidence and mortality worldwide: sources, methods and major patterns in GLOBOCAN 2012. Int J. Cancer 136, E359-E386 (2015)

2. Pernar, C. H., Ebot, E. M., Wilson, K. M. \& Mucci, L. A. The epidemiology of prostate cancer. Cold Spring Harb. Perspect. Med. 12, 8 (2018).

3. Attard, G. et al. Prostate cancer. Lancet 387, 70-82 (2016).
4. Kgatle, M. M., Kalla, A. A., Islam, M. M., Sathekge, M. \& Moorad, R. Prostate cancer: epigenetic alterations, risk factors, and therapy. Prostate Cancer 2016, 5653862 (2016).

5. Sartor, A. O. Progression of metastatic castrate-resistant prostate cancer: impact of therapeutic intervention in the post-docetaxel space. J. Hematol. Oncol. 4, 18 (2011).

6. Ohshio, Y. et al. Cancer-associated fibroblast-targeted strategy enhances antitumor immune responses in dendritic cell-based vaccine. Cancer Sci. 106, 134-142 (2015)

7. Harper, J. \& Sainson, R. C. Regulation of the anti-tumour immune response by cancer-associated fibroblasts. Semin. Cancer Biol. 25, 69-77 (2014).

8. Herrera, M. et al. Differential distribution and enrichment of non-coding RNAs in exosomes from normal and Cancer-associated fibroblasts in colorectal cancer. Mol. Cancer 17, 114 (2018).

9. Arscott, W. T. et al. lonizing radiation and glioblastoma exosomes: implications in tumor biology and cell migration. Transl. Oncol. 6, 638-648 (2013).

10. Duijvesz, D., Luider, T., Bangma, C. H. \& Jenster, G. Exosomes as biomarker treasure chests for prostate cancer. Eur. Urol. 59, 823-831 (2011).

11. Lasser, C. \& Exosomal, R. N. A. as biomarkers and the therapeutic potential of exosome vectors. Expert Opin. Biol. Ther. 12(Suppl 1), S189-S197 (2012).

12. Kim, W. T. \& Kim, W. J. MicroRNAs in prostate cancer. Prostate Int. 1, 3-9 (2013).

13. Hessvik, N. P., Sandvig, K. \& Llorente, A. Exosomal miRNAs as biomarkers for prostate cancer. Front. Genet. 4, 36 (2013).

14. Zhang, W. et al. Identification of candidate miRNA biomarkers from miRNA regulatory network with application to prostate cancer. J. Transl. Med. 12, 66 (2014).

15. Li, S. et al. miR-423-5p contributes to a malignant phenotype and temozolomide chemoresistance in glioblastomas. Neuro Oncol. 19, 55-65 (2017).

16. Stiuso, P. et al. MicroRNA-423-5p promotes autophagy in cancer cells and is increased in serum from hepatocarcinoma patients treated with sorafenib. Mol. Ther. Nucleic Acids 4, e233 (2015).

17. Yang, $\mathrm{H}$. et al. Exosomal miR-423-5p targets SUFU to promote cancer growth and metastasis and serves as a novel marker for gastric cancer. Mol. Carcinog. 57, 1223-1236 (2018)

18. Nolan, K. et al. Structure of Gremlin-2 in complex with GDF5 gives insight into DAN-family-mediated BMP antagonism. Cell Rep. 16, 2077-2086 (2016).

19. Rojo, A. I. et al. Signaling pathways activated by the phytochemical nordihydroguaiaretic acid contribute to a Keap1-independent regulation of Nrf2 stability: Role of glycogen synthase kinase-3. Free Radic. Biol. Med. 52, 473-487 (2012)

20. Malik, D., Kaul, D., Chauhan, N. \& Marwaha, R. K. miR-2909-mediated regulation of KLF4: a novel molecular mechanism for differentiating between B-cell and T-cell pediatric acute lymphoblastic leukemias. Mol. Cancer 13, 175 (2014).

21. Dong, Y. et al. IncRNA Gm15290 promotes cell proliferation and invasion in lung cancer through directly interacting with and suppressing the tumor suppressor miR-615-5p. Biosci. Rep. 38, BSR20181150 (2018).

22. Li, Y. Y. et al. Cancer-associated fibroblasts contribute to oral cancer cells proliferation and metastasis via exosome-mediated paracrine miR-34a-5p. EBioMedicine 36, 209-220 (2018)

23. Calon, A., Tauriello, D. V. \& Batlle, E. TGF-beta in CAF-mediated tumor growth and metastasis. Semin. Cancer Biol. 25, 15-22 (2014).

24. Shi, L. et al. Combination therapy of TGF-beta blockade and commensalderived probiotics provides enhanced antitumor Immune response and tumor suppression. Theranostics 9, 4115-4129 (2019).

25. Ren, J. et al. Carcinoma-associated fibroblasts promote the stemness and chemoresistance of colorectal cancer by transferring exosomal IncRNA H19. Theranostics 8, 3932-3948 (2018).

26. Bray, F. et al. Global cancer statistics 2018: GLOBOCAN estimates of incidence and mortality worldwide for 36 cancers in 185 countries. CA Cancer J. Clin. 68, 394-424 (2018).

27. Rana, S., Malinowska, K. \& Zoller, M. Exosomal tumor microRNA modulates premetastatic organ cells. Neoplasia 15, 281-295 (2013).

28. Yang, W. W. et al. Epiregulin promotes lung metastasis of salivary adenoid cystic carcinoma. Theranostics 7, 3700-3714 (2017).

29. Goldraich, L. A. et al. Transcoronary gradient of plasma microRNA 423-5p in heart failure: evidence of altered myocardial expression. Biomarkers 19 135-141 (2014).

30. Nabialek, E. et al. Circulating microRNAs (miR-423-5p, miR-208a and miR-1) in acute myocardial infarction and stable coronary heart disease. Minerva Cardioangiol. 61, 627-637 (2013). 
31. Lin, H. et al. Inhibition of miR-423-5p suppressed prostate cancer through targeting GRIM-19. Gene 688, 93-97 (2019).

32. Wu, Q., Tang, S. G. \& Yuan, Z. M. Gremlin 2 inhibits adipocyte differentiation through activation of Wnt/beta-catenin signaling. Mol. Med. Rep. 12 5891-5896 (2015).

33. Abulaiti, A. et al. Interaction between non-small-cell lung cancer cells and fibroblasts via enhancement of TGF-beta signaling by IL-6. Lung Cancer 82, 204-213 (2013).

34. Gao, M. Q. et al. Human breast cancer-associated fibroblasts enhance cancer cell proliferation through increased TGF-alpha cleavage by ADAM17. Cancer Lett. 336, 240-246 (2013).

35. Cai, J. et al. Fibroblasts in omentum activated by tumor cells promote ovarian cancer growth, adhesion and invasiveness. Carcinogenesis $\mathbf{3 3}$ 20-29 (2012).

36. Shedden, K., Xie, X. T., Chandaroy, P., Chang, Y. T. \& Rosania, G. R. Expulsion of small molecules in vesicles shed by cancer cells: association with gene expression and chemosensitivity profiles. Cancer Res. $\mathbf{6 3}$ 4331-4337 (2003).

37. Boelens, M. C. et al. Exosome transfer from stromal to breast cancer cells regulates therapy resistance pathways. Cell 159, 499-513 (2014).

38. $\mathrm{Hu}, \mathrm{Y}$. et al. Fibroblast-derived exosomes contribute to chemoresistance through priming cancer stem cells in colorectal cancer. PLOS ONE 10, e0125625 (2015).
39. Luga, V. et al. Exosomes mediate stromal mobilization of autocrine Wnt-PCP signaling in breast cancer cell migration. Cell 151, 1542-1556 (2012).

40. Albrengues, J. et al. Epigenetic switch drives the conversion of fibroblasts into proinvasive cancer-associated fibroblasts. Nat. Commun. 6, 10204 (2015).

41. Calvo, F. et al. Mechanotransduction and YAP-dependent matrix remodelling is required for the generation and maintenance of cancer-associated fibroblasts. Nat. Cell Biol. 15, 637-646 (2013).

42. Procopio, M. G. et al. Combined CSL and p53 downregulation promotes cancer-associated fibroblast activation. Nat. Cell Biol. 17, 1193-1204 (2015).

43. Webber, J., Steadman, R., Mason, M. D., Tabi, Z. \& Clayton, A. Cancer exosomes trigger fibroblast to myofibroblast differentiation. Cancer Res. 70, 9621-9630 (2010).

44. Webber, J. P. et al. Differentiation of tumour-promoting stromal myofibroblasts by cancer exosomes. Oncogene 34, 290-302 (2015).

45. Josson, S. et al. Stromal fibroblast-derived miR-409 promotes epithelial-tomesenchymal transition and prostate tumorigenesis. Oncogene 34, 2690-2699 (2015).

46. Li, J. et al. Exosome-derived microRNAs contribute to prostate cancer chemoresistance. Int J. Oncol. 49, 838-846 (2016).

47. Dauer, P. et al. Inactivation of cancer-associated-fibroblasts disrupts oncogenic signaling in pancreatic cancer cells and promotes its regression. Cancer Res. 78, 1321-1333 (2018). 